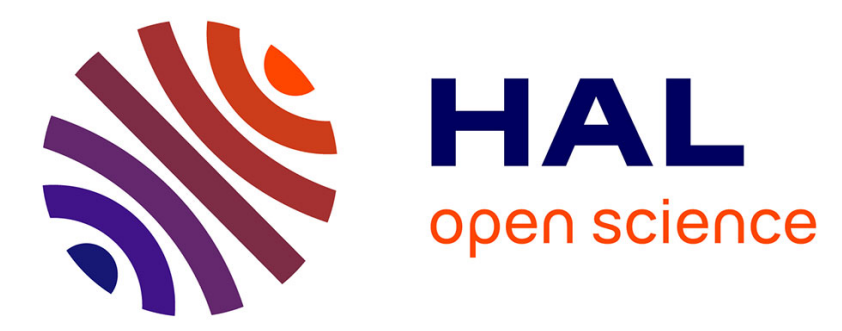

\title{
Metrical Rhythm Implicitly Orients Attention in Time as Indexed by Improved Target Detection and Left Inferior Parietal Activation
}

\author{
Deirdre Bolger, Jennifer T Coull, Daniele Schön
}

\section{- To cite this version:}

Deirdre Bolger, Jennifer T Coull, Daniele Schön. Metrical Rhythm Implicitly Orients Attention in Time as Indexed by Improved Target Detection and Left Inferior Parietal Activation. Journal of Cognitive Neuroscience, 2014, 26 (3), pp.593-605. 10.1162/jocn_a_00511 . hal-02446661

\author{
HAL Id: hal-02446661 \\ https://hal.science/hal-02446661
}

Submitted on 21 Jan 2020

HAL is a multi-disciplinary open access archive for the deposit and dissemination of scientific research documents, whether they are published or not. The documents may come from teaching and research institutions in France or abroad, or from public or private research centers.
L'archive ouverte pluridisciplinaire HAL, est destinée au dépôt et à la diffusion de documents scientifiques de niveau recherche, publiés ou non, émanant des établissements d'enseignement et de recherche français ou étrangers, des laboratoires publics ou privés. 


\title{
Metrical Rhythm Implicitly Orients Attention in Time as Indexed by Improved Target Detection and Left Inferior Parietal Activation
}

\author{
Deirdre Bolger ${ }^{1}$, Jennifer T. Coull ${ }^{2}$, and Daniele Schönn ${ }^{1,3}$
}

\begin{abstract}
When we direct attentional resources to a certain point in time, expectation and preparedness is heightened and behavior is, as a result, more efficient. This future-oriented attending can be guided either voluntarily, by externally defined cues, or implicitly, by perceived temporal regularities. Inspired by dynamic attending theory, our aim was to study the extent to which metrical structure, with its beats of greater or lesser relative strength, modulates attention implicitly over time and to uncover the neural circuits underlying this process of dynamic attending. We used fMRI to investigate whether auditory meter generated temporal expectancies and, consequently, how it affected processing of auditory and visual targets. Participants listened to a continuous auditory metrical sequence and pressed a button whenever an auditory or visual target was presented.
\end{abstract}

\section{INTRODUCTION}

Focusing (or "orienting") attention to future points in time prepares us for effective action. The more reliably we can anticipate this moment in time, the better we can focus our attention toward it and, as a result, the more effective our behavior at this moment is likely to be. Extensive research has demonstrated that facilitating temporal prediction by providing reliable cues as to when something will happen facilitates processing of this event in a twofold manner: First, there is an enhancement of the sensory processing of stimuli occurring at this time (Correa, Lupiáñez, \& Tudela, 2005; Martens \& Johnson, 2005; Barnes \& Jones, 2000), and second, motor responses to these stimuli are facilitated (Coull \& Nobre, 1998; Niemi \& Naataanen, 1981; Woodrow, 1914). According to Jones (1976), "we are creatures of rhythm," and we readily use the regularity and predictability of rhythmic presentation to anticipate future events. Furthermore, the hierarchical structure of rhythm permits us to do this on several ratio-related timescales. When tapping along to a beat, we are able to change the periodicity of our tapping to emphasize a different ratio-related time interval (e.g.,

\footnotetext{
${ }^{1}$ Aix-Marseille Université, INS, ${ }^{2}$ Aix-Marseille Université-CNRS, LNC, ${ }^{3}$ INSERM, U1106, Marseille, France
}

The independent variable was the time of target presentation with respect to the metrical structure of the sequence. Participants' RTs to targets occurring on strong metrical positions were significantly faster than responses to events falling on weak metrical positions. Events falling on strong beats were accompanied by increased activation of the left inferior parietal cortex, a region crucial for orienting attention in time, and, by greater functional connectivity between the left inferior parietal cortex and the visual and auditory cortices, the SMA and the cerebellum. These results support the predictions of the dynamic attending theory that metrical structure with its relative strong and weak beats modulates attentional resources over time and, in turn, affects the functioning of both perceptual and motor preparatory systems.

we could tap to every two tones or to every three). This demonstrates that we have access to a general temporal structure composed of multiple hierarchically related periodicities or, in other words, the meter. An important implication of meter's hierarchical structure is that not all beats are perceived as equally salient, leading to the perception of stronger and weaker beats within a meter and, consequently, events of greater and lesser significance (Large \& Palmer, 2002; Parncutt, 1994).

The dynamic attending theory (DAT; Large \& Jones, 1999; Jones \& Boltz, 1989; Jones, Boltz, \& Kidd, 1982) emphasizes the role of metrical structure in facilitating future-oriented attending through a coordination of our internal neural oscillations with periodicities at each hierarchical level of an external temporal structure. This cocoordinative process is known as entrainment (Drake, Penel, \& Bigand, 2000; Large, 2000). A computational implementation of DAT (Large \& Jones, 1999) predicts that metrical structure modulates attention toward weaker or stronger beats, such that events coinciding with strong beats are attributed more attentional energy and, thus, are more highly anticipated. Behavioral studies have provided strong support for a role of meter in generating expectations and orienting attention to specific points in time. Stimulus processing and reactivity have been found to be enhanced for events forming part of a regular, 
rhythmic sequence compared with those forming part of an irregular sequence (Jones \& Yee, 1997; Klein \& Jones, 1996), and listeners are more accurate in judging the pitch of tones that are rhythmically expected compared with those that are rhythmically unexpected (Jones et al., 2002; Jones \& Ralston, 1991). Moreover, in a prior behavioral study of our own, we found that RTs to stimuli presented on the strongest metrical position of either an isochronous meter or an extract of classical music were consistently faster than those on the weakest, or even intermediate, positions, with this effect being independent of stimulus modality (Bolger, Trost, \& Schön, 2013). Collectively, these behavioral results indicate that attention is preferentially oriented in time toward strong or expected beats.

Rhythmically defined temporal expectations can be thought of as an example of exogenous or stimulus-driven orienting of attention in time (Barnes \& Jones, 2000; Coull \& Nobre, 1998). In exogenous temporal orienting, the generation of expectations for a particular moment in time is driven by perceived regularities inherent in the time structure of the stimulus (such as a piece of music) and can occur automatically, in the absence of strategic or voluntary mechanisms (Rohenkohl, Coull, \& Nobre, 2011; Barnes \& Jones, 2000). On the other hand, endogenous orienting of attention is initiated by a symbolic temporal cue (such as a red traffic light), which in turn leads one to voluntarily generate internal predictions regarding when the anticipated event will occur (Coull \& Nobre, 1998). Despite the functional differences between endogenous and exogenous orienting of attention (Rohenkohl et al., 2011), the question of whether these two mechanisms are driven by distinct or by common, neural circuits is not yet resolved. fMRI studies have consistently identified a role for left inferior parietal cortex (IPC; BA 40) and left intraparietal sulcus in both endogenous (Coull, Frith, Büchel, \& Nobre, 2000; Coull \& Nobre, 1998) and exogenous (Coull, Vidal, Goulon, Nazarian, \& Craig, 2008; Assmus et al., 2003; Dreher, Koechlin, Ali, \& Grafman, 2002; Sakai et al., 2000) forms of temporal orienting (Wiener, Turkeltaub, \& Coslett, 2011). These initial findings suggest a strong motor component to temporal orienting (Nobre, 2001; Coull et al., 2000) as the left IPC has also been implicated in motor attention (Rushworth, Nixon, Renowden, Wade, \& Passingham, 1997), the anticipation of a specific type of movement before its execution. However, endogenous temporal orienting has since been shown to activate left intraparietal sulcus independently of the laterality or type of motor effector used to register the response (Cotti, Rohenkohl, Stokes, Nobre, \& Coull, 2011) or by a purely perceptual task that has no motor goal (Davranche, Nazarian, Vidal, \& Coull, 2011), strongly suggesting that left parietal cortex represents a fundamental, context-independent neuroanatomical substrate for the orienting of attention in time. Yet such contextindependent parietal activity may be accompanied by context-dependent activity in other, more stimulus-specific areas. For example, temporally predictable visual stimuli increase activity in early visual processing areas (Davranche et al., 2011; Bueti, Bahrami, Walsh, \& Rees, 2010) and modulate electrophysiological markers of visual attention (Rimmele, Jolsvai, \& Sussman, 2011; Rohenkohl \& Nobre, 2011; Correa, Lupiáñez, Madrid, \& Tudela, 2006; Praamstra, Kourtis, Kwok, \& Oostenveld, 2006). Analogously, temporally predictable auditory or tactile stimuli have been shown to enhance electrophysiological markers of auditory or somatosensory attention, respectively (Lange \& Röder, 2006).

On the other hand, neuroimaging studies of rhythm (e.g., Bengtsson et al., 2009; Karabanov, Blom, Forsman, \& Ullén, 2009; Chen, Zatorre, \& Penhune, 2006; Jäncke, Loose, Lutz, Specht, \& Shah, 2000; Rao et al., 1997) have implicated a different set of motor regions, including premotor cortex, SMA, and cerebellum, even when tasks did not require a timed motor response (Chen, Penhune, \& Zatorre, 2008; Schubotz, Friederici, \& von Cramon, 2000). More specifically, the perception of a metrical beat within a complex rhythmic sequence activates SMA and BG (Kung, Chen, Zatorre, \& Penhune, 2013; Grahn, Henry, \& McAuley, 2011; Grahn \& Brett, 2007; Bengtsson, Ehrsson, Forssberg, \& Ullén, 2005; Lewis, Wing, Pope, Praamstra, \& Miall, 2004) as well as reinforcing their functional interconnectivity (Grahn \& Rowe, 2009). Although these studies identify areas implicated in the fundamental process of perceiving rhythm or temporal structure, it is also possible to identify areas implicated in using the temporal predictability of the rhythmic structure to enhance information processing (as in the temporal orienting studies reviewed previously). Very recently, Geiser, Notter, and Gabrieli (2012) found that the behavioral improvement in (nontemporal) auditory discrimination induced by presenting stimuli in a temporally predictable, rhythmic (isochronous) sequence was accompanied by increased activity in the BG (specifically the putamen). Similarly, Marchant and Driver (2012) found that the improvement in visual target detection induced by temporally regular, rather than irregular, stimulus presentation was also accompanied by increased activity in the BG, as well as in bilateral pFC, insula, and left IPC. However, in both of these studies, rhythmic or temporally regular sequences were compared with temporally irregular sequences. We aim to extend and refine this literature and to improve the temporal resolution of rhythm-induced facilitation by comparing regional brain activity for individual elements within a beat-based sequence. Critically, all experimental conditions used in our experiment were equally rhythmic to control for processes related to the perception of rhythm per se. By identifying regions preferentially activated by temporally attended (strong) versus less attended (weak) beats within a sequence, we hope to build a bridge between the beat perception and temporal attentional orienting literature.

During scanning, participants performed a speeded RT task while listening to a repeating, eight-tone isochronous rhythm, which continued uninterrupted throughout each 
session; this background rhythm will be referred to as the entraining sequence. The target stimuli, either auditory or visual, were presented randomly at one of four selected metrical positions of the entraining sequence. These positions differed in metrical strength: for example, the first, on-beat tone had a strong metrical position whereas the fourth, off-beat tone had a weak position. From a behavioral point of view, we aimed to confirm the results of an earlier behavioral study using the same task, which showed enhanced performance for targets presented on strong versus weak metrical positions, thereby providing evidence for enhanced temporal attention at strong metrical positions. From a neural point of view, we asked whether fMRI data would support this behavioral result by revealing greater activation of cortical areas implicated in the temporal orienting of attention for targets presented at strong, rather than weak, metrical positions. On the basis of prior fMRI studies of temporal orienting (e.g., Coull \& Nobre, 1998, 2008), we hypothesized that left inferior parietal activity would increase in response to targets presented at strong metrical positions. In addition, as our entraining sequence has a very regular and simple rhythmic structure from which the beat should be easily induced, we investigated whether activity in SMA and BG would be modulated as a function of metrical position. On the basis of prior fMRI studies of rhythm and beat perception (e.g., Grahn \& Brett, 2007; Bengtsson et al., 2005), we hypothesized that activity in these areas would be greater for targets presented at strong rather than weak metrical positions. Finally, if temporal orienting of attention facilitates early perceptual processing, we would predict increased activity in auditory (or visual) processing brain areas when auditory (or visual) target stimuli were presented on a strong, rather than weak, metrical positions. Importantly, based on prior behavioral (Bolger et al., 2013; Escoffier, Yeo, \& Schirmer, 2010), electrophysiological (Besle, Hussain, Giard, \& Bertrand, 2013; Lange \& Röder, 2006), and neuroimaging evidence (Grahn et al., 2011; Bueti \& Macaluso, 2010), this effect should be cross-modal.

\section{METHODS}

\section{Participants}

Seventeen healthy volunteers participated in the study, nine of whom were women and two were left-handed (mean age $=26.75$ years, ranging from 19 to 40 years). One participant was a musician, and all others were considered as nonmusicians, either having no formal musical training or less than 24 months of musical training in the previous 10 years. The experimental protocol was approved by the local ethics committee, and written informed consent was obtained from all participants before the experiment. Data from three additional participants were acquired but had to be discarded because of the presence of significant artifacts in their images caused by movement during scanning. Participants were paid 30 Euros for their participation.

\section{Cognitive Task}

Participants performed a timed motor detection task in the presence of a repeating, isochronous auditory rhythm, which we refer to here as the metronome. The metronome was composed of repetitions of a basic eight-tone pattern; the tones were sinusoids, the first tone of each basic pattern had a frequency of $880 \mathrm{~Hz}$, whereas the remaining seven tones had a frequency of $440 \mathrm{~Hz}$. Each sinusoidal tone lasted $100 \mathrm{msec}$, and tones were presented with an SOA of $250 \mathrm{msec}$. A single eighttone pattern therefore had a duration of $2000 \mathrm{msec}$, and this basic pattern was repeated continuously and seamlessly throughout the experiment. The target stimuli to be detected were either auditory or visual. The auditory target was a shaker sound (duration, $82 \mathrm{msec}$ ), which was generated using the Csound sound synthesis software (Vercoe, MIT Labs, 1985). Its random spectral structure and, therefore, noise-like sound quality meant that it was easily discriminable from the sinusoidal tones comprising the metronome. The visual target was an "X," which was presented by means of light-emitting diodes (green LEDs) mounted on a specially designed board that was positioned at the end of the fMRI machine bore. The LED lights were made visible to the participants during scanning by means of a mirror mounted at eye level and resulted in a stimulus with approximately $2^{\circ}$ of field of view. Stimuli presentation as well as synchronization with EPI acquisition was controlled via National Instruments cards and Labview custom programming allowing for a timing accuracy at the millisecond level.

The experimental variable was the moment of presentation of a target stimulus within the basic eight-tone pattern of the metronome, which we refer to here as the metrical position (MP). We presumed that the metronome induces a binary meter with a strong beat coinciding with the first tone and a weaker beat coinciding with the fifth tone (see Figure 1). Thus, two MPs (MP1 and MP5) were selected on the basis of their relative metrical strength and, consequently, the relative levels of expectation that may be attributed to a target occurring at these positions (Essens \& Povel, 1985). Moreover, to add temporal variability, we added two further positions, MP4 and MP8, which immediately preceded (by $250 \mathrm{msec}$ ) the weak (MP5) and strong (MP1) beats, respectively. These positions were chosen on the basis of a prior behavioral study (Bolger et al., 2013), showing that RTs to targets presented just before metrically weak or strong beats were comparable with those presented on the weak or strong beats, respectively (strong and weak metrical "windows"). Participants were asked to react as quickly as possible to the presentation of a stimulus by pressing a response button in their right hand. 
Figure 1. The attentional curve, representing the variation in attention level as a function of metrical structure, obtained using one oscillator whose periods was $2000 \mathrm{msec}$. Gray areas illustrate strong (MP1 and MP8) and weak (MP4 and MP5) metrical zones as predicted on the basis of our previous work (Bolger et al., 2013). Auditory or visual target items could be presented at one of the four metrical positions with a minimum SOA of $2 \mathrm{sec}$. At the bottom, a music notation representation of the entraining metronome and two target visual stimuli $(\mathrm{X})$.

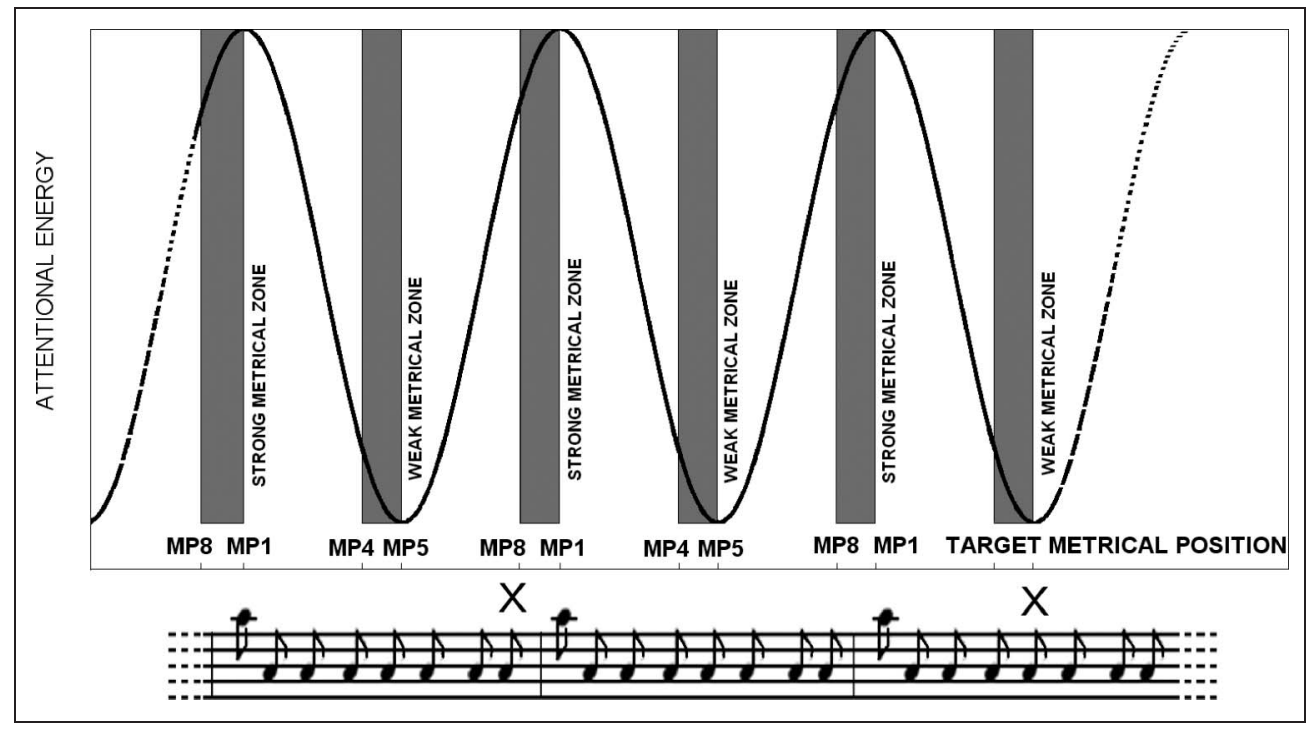

The fMRI experimental design was event-related. For each participant, there were four experimental runs, two in which the targets were auditory (auditory runs) and two in which visual targets were presented (visual runs). The auditory metronome was presented continuously during each of the four scanning runs. The order of the runs (auditory or visual) was counterbalanced across participants so that half of the participants began with the two auditory runs followed by the two visual runs. Each scanning run lasted $7 \mathrm{~min}$, and during each, participants were presented with 24 targets per experimental condition (MP1, MP4, MP5, or MP8). The four experimental conditions were pseudorandomly distributed throughout each run using a nonaging distribution, with the additional constraint that the minimum target onset asynchrony was $2 \mathrm{sec}$, thus ensuring that two targets were never presented within a single eight-tone measure. Before scanning, participants were asked to perform a short training session of both the auditory and visual detection tasks using a computer-based simulation of the experiment implemented using Presentation software (Presentation 14.5, Neurobehavioral Systems, Albany, CA).

\section{fMRI Scanning}

Scanning was carried out using a 3T (3T-MAGNEX, Ettlingen, Germany) Bruker Medspec 30/80 advanced whole body MRI system, equipped with head coil. EPI was used to acquire an interleaved sequence of T2*-weighted images in the axial plan. The volume of the acquired image was composed of $35 \times 3 \mathrm{~mm}$ slices with an interscan interval (repetition time) of $2.33 \mathrm{sec}$. For each participant, four scanning runs were carried out (approximately 7 min per run), and 192 image volumes were acquired for each run. The size of the image volume was chosen to permit scanning of the entire cerebral cortex and the cerebellum. The auditory stimuli (metronome and auditory targets) were presented via headphones (MR-Confon), and attenuation of the scanner noise was achieved by the use of earplugs, which all participants used. To further attenuate scanner noise, the bore of the scanner was lined with insulating foam. A foam pad was also used to support the back of the head and to cover the ears, holding the headphones in place and providing extra protection against the scanner noise. Participants were instructed to remain as still as possible during scanning, avoiding all movement other than to respond to targets by button press, which was always held in the right hand and which they pushed using their right thumb. To ensure that participants were as comfortable as possible, a foam pad also supported their legs. In the case of emergency or discomfort, participants had a panic button in their left hand. A structural MRI was acquired using a standard T1-weighted scanning sequence of $1 \mathrm{~mm}^{3}$ resolution. Fieldmaps were also collected to improve coregistration between the EPI images and the anatomical image (Hutton et al., 2002) and to reduce the effects of spatial distortion because of magnetic field inhomogeneity.

\section{Data Analysis}

The functional images were processed and analyzed using Statistical Parametric Mapping (SPM8). Slice timing correction was carried out on all functional images, and using the SPM fieldmap toolbox (Andersson, Hutton, Ashburner, Turner, \& Friston, 2001), the acquired fieldmaps were employed to undistort the EPI images. The undistorted fMRI data were motion corrected with respect to the first volume of the first session using the SPM realignment tool. All images were spatially normalized using DARTEL, an algorithm for diffeomorphic image registration (Ashburner, 2007) by matching each to a custom-built group template generated using the DARTEL tool of the SPM8 package. To generate the template, first the structural T1-weighted 
scans of all 17 participants were segmented into different tissue types, gray and white matter, using the SPM8 segmentation tool, and the initial template for DARTEL registration was created by generating intensity averages of these gray and white matter images. This initial smooth template was then sharpened iteratively after each phase of registration. Following normalization, the fMRI data were spatially smoothed by convoluting it with Gaussian kernels of $8 \mathrm{~mm}$ width.

The experimental factors of metrical position (weak/ strong) and target modality (auditory/visual) comprised a $2 \times 2$ factorial design. Their effects on regional brain activity were estimated according to a general linear model in voxel brain space. First-level analyses were carried out separately for all participants. For each participant, four event-related regressors of null duration, time-locked to the target onset, were defined that captured the four possible combinations of the two experimental factors: Auditory Weak, Auditory Strong, Visual Weak, Visual Strong. The "Auditory" regressors modeled targets presented in the auditory modality (the shaker sound), whereas the "Visual" regressors modeled targets presented in the visual modality (the "X"). The "Strong" regressors modeled targets presented on either MP1 or MP8, whereas the "Weak" regressors modeled targets presented on either MP4 or MP5. Behavioral data collected before (see Bolger et al., 2013) and during scanning showed that, for both target modalities, RTs to targets at MP1 and MP8 showed a high degree of similarity and were significantly faster than those to targets at MP4 and MP5. Because faster RTs reflect increased expectancies (Nobre \& Coull, 2010), we grouped together trials in which the target appeared at either MP1 or MP8 as the "Strong" condition, whereas trials in which the target appeared at MP4 or MP5 were grouped together as the "Weak" condition.

We carried out an analysis of the main effect of target modality to verify that participants were processing the target stimuli in an expected manner. To this end, we compared Auditory to Visual conditions, averaging together Strong and Weak target types (i.e., auditory target activity was defined by [AuditoryWeak + AuditoryStrong] [VisualWeak + VisualStrong], and visual target activity was defined by [VisualWeak + VisualStrong] - [AuditoryWeak + AuditoryStrong]). To investigate the main effect of metrical position, we compared Strong to Weak conditions, averaging across modalities (i.e., strong temporal expectancy was defined by [AuditoryStrong + VisualStrong] [AuditoryWeak + VisualWeak], whereas weak expectancy was defined by [AuditoryWeak + VisualWeak] - [AuditoryStrong + VisualStrong]). Note that the participant's task of detecting targets remains the same across conditions, meaning that any activity related simply to perceiving, and responding to, targets is cancelled out.

Although behavioral data showed no significant interaction between target modality and metrical position (see also Bolger et al., 2013), we wished to examine whether brain activity was differentially activated by tem- poral expectation across the two modalities. Therefore, to compare the effects of metrical position across the two modalities (i.e., Modality $\times$ Meter interaction), we first specified the difference between Strong versus Weak expectancies for the Auditory and Visual modalities individually and then compared them directly (i.e., areas activated more by strong expectancies in the auditory, rather than visual, modality were identified by [AuditoryStrong AuditoryWeak] - [VisualStrong - VisualWeak], whereas those preferentially activated by strong expectancies in the visual modality were identified by [VisualStrong VisualWeak] - [AuditoryStrong - AuditoryWeak]). Each contrast was specified for every participant; thus, for each contrast, 17 SPMs were generated.

For each of the contrasts specified, the SPMs generated for each participant were entered into a second-level, random effects analysis implementing one-sample $t$ tests. We adopted a significance threshold for whole-brain analysis of $p<.05$, family-wise error (FWE) corrected for multiple comparisons, except for areas for which we had a strong a priori hypothesis based on previous studies. For the effects of target modality, these hypothesized areas comprised primary visual and auditory cortices. For the effects of metrical position, hypothesized areas comprised SMA and BG, previously implicated in rhythm perception (Grahn \& McAuley, 2009; Grahn \& Rowe, 2009; Chen et al., 2006; Bengtsson et al., 2005; Schubotz et al., 2000; Grahn \& Brett, 1997), and left IPC and cerebellum, previously implicated in temporal expectation (Coull, Davranche, Nazarian, \& Vidal, 2013; Cotti et al., 2011; Davranche et al., 2011; Wiener et al., 2011; Geiser, Zaehle, Jancke, \& Meyer, 2008; Coull \& Nobre, 1998). Hypothesized areas were tested using small volume correction (SVC) analyses, thresholded at $p<.05$, FWE corrected for multiple comparisons. The volumes of interest (VOIs) used to perform the SVC were derived from the SPM anatomical automatic labeling toolbox (Tzourio-Mazoyer et al., 2002) and comprised (i) Heschl's gyrus and planum temporal, (ii) inferior occipital cortex and fusiform gyrus, (iii) SMA, (iv) BG (putamen and caudate), (v) left IPC, and (vi) cerebellum.

\section{Psychophysiological Interaction Analysis}

We investigated the presence of changes in functional connectivity as a function of the metrical position of the target using psychophysiological interaction (PPI) analysis (Büchel \& Friston, 2002). The PPI analysis allowed us to investigate whether region-to-region coactivation was modulated as a function of temporal expectation (as indexed by the metrical position of the target) using the auditory and visual target data averaged together (to increase statistical power), although we tested modalities separately when investigating sensory regions (visual and auditory cortices). The seed region for our PPI analysis was the left IPC region from the Strong-Weak contrast ([-46 -50 -44], MNI coordinates). Thus, our 
physiological regressor was activity in the specified left IPC cluster, our psychological regressor was metrical position, and the resulting PPI represented the interaction of the physiological and psychological regressors.

We extracted the first eigenvariate time series from the individual participant data, thus creating a physiological regressor for each run. This extracted data were multiplied by the psychological regressor and entered into the PPI analyses to generate PPI maps for each individual participant. The individual, participant-level PPI maps were then entered into a second-level, random effects analysis so that changes in connectivity of the left IPC as a function of the metrical position of the target stimulus (i.e., the strength of temporal expectation) could be investigated. In detailing our results, we report regions that survive a significance threshold of $p<.05$, corrected for multiple comparisons (FWE), after SVC in hypothesized VOIs (see above for details of the six VOIs interrogated).

\section{RESULTS}

\section{Behavioral Data}

A $2 \times 4$ repeated-measures ANOVA was carried out for the factors Target Modality (auditory/visual) and Metrical Position (MP1, MP4, MP5, or MP8). We found a significant effect of Metrical Position on performance, $F(3,48)=32.8$, $p<.0001$. Post hoc analyses showed that, in the case of both auditory and visual targets, RTs to targets presented at MP1 and MP8 did not differ significantly, and this was also the case for RTs to targets presented at MP4 and MP5. Furthermore, for both modalities, RTs to targets at both MP1 and MP8 differed significantly from those at
MP4 and MP5 (always $p<.01$ ). There was no main effect of Target Modality on performance, $F(1,16)=1.5329, p<$ .236, nor was there a significant interaction between Target Modality and Metrical Position, $F(3,48)=2.344, p<.08$, although results suggest a trend for metrical position effects to be stronger in the auditory modality than in the visual one.

\section{fMRI Data}

\section{Main Effect of Target Modality}

To verify that the participants were processing the auditory and visual target stimuli in an expected manner, we carried out an analysis of the main effect of Target Modality, averaged across Strong and Weak conditions. The Auditory-Visual contrast revealed significant activation in auditory cortex bilaterally, whereas the Visual-Auditory contrast identified significant activations in primary and secondary visual cortex, comprising V1, V2, and V4 bilaterally (Table 1 ).

\section{Main Effect of Metrical Position}

An analysis of the Strong versus Weak conditions, averaged across Auditory and Visual modalities, revealed a maximum activation in the area of the left IPC ([-46, -50 , $44], Z=5.01 ; p=.001$, small volume corrected, .05 FWE whole brain) during the strong metrical condition (Figure 3). No other areas survived the correction for multiple comparisons, either at the whole-brain or VOI level.

To gain a better insight into the relative effect size for each of the four metrical positions (MP1, MP4, MP5, MP8) and the relative contribution of each to the activity observed at the region maximally implicated in the

Table 1. Areas of Activation for Analyses of Main Effects of Modality and Meter

\begin{tabular}{|c|c|c|c|c|c|c|}
\hline Region & $x$ & $y$ & $z$ & $B A$ & z Score & $p_{\text {corrected }}$ \\
\hline \multicolumn{7}{|c|}{ Main Effect of Modality for VOI Analysis } \\
\hline \multicolumn{7}{|l|}{ Auditory-Visual } \\
\hline \multirow[t]{2}{*}{ Right auditory } & 54 & -24 & 8 & BA 41, BA 22 & 4.22 & .003 \\
\hline & 44 & -38 & 14 & & 3.45 & \\
\hline \multirow[t]{2}{*}{ Left auditory } & -57 & -29 & 11 & BA 22 & 3.04 & .001 uncorr \\
\hline & -44 & -35 & 9 & & & \\
\hline \multicolumn{7}{|l|}{ Visual-Auditory } \\
\hline Right visual & 35 & -69 & -12 & BA 18, BA 19, BA 37 & 4.39 & .02 \\
\hline Left visual & -32 & -62 & -18 & BA 18, BA 19, BA 37 & 5.58 & $<.001^{\mathrm{a}}$ \\
\hline \multicolumn{7}{|c|}{ Main Effect of Meter for VOI Analysis } \\
\hline Left inferior parietal & -46 & -50 & 44 & BA 40 & 4.92 & .001 \\
\hline
\end{tabular}

${ }^{\text {a }}$ Surviving FWE $(p<.05)$ at whole-brain level. 


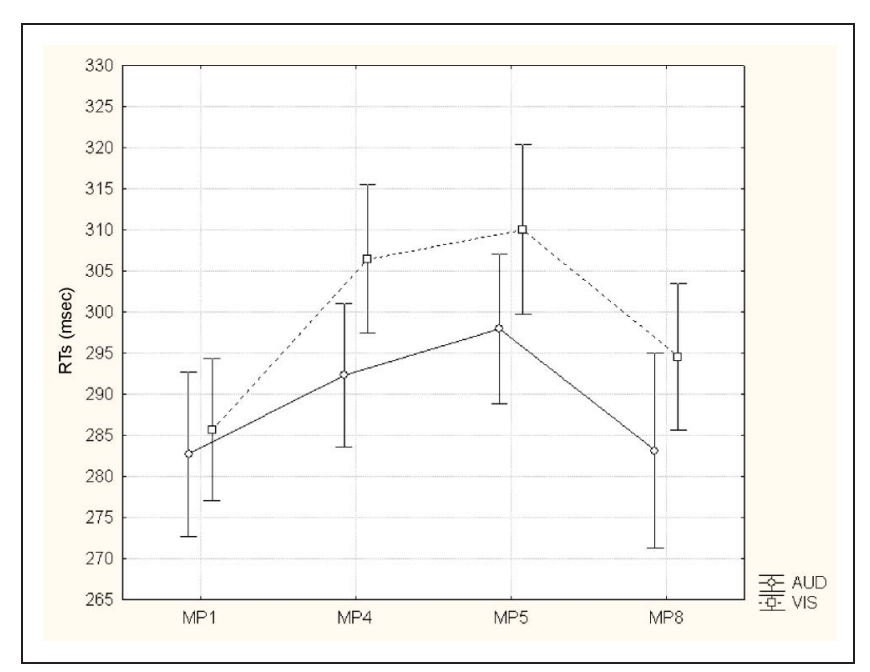

Figure 2. RTs (in msec) to targets presented in the four different metrical positions (strong: MP1 and MP8 and weak: MP1 and MP8) averaged across participants in the auditory and visual detection tasks.

main effect of Metrical Position, we extracted the beta values from the peak left IPC voxel $[-46-5044]$ from the Strong minus Weak contrast separately for each participant, modality, and for each of the four metrical positions. The extracted beta values were normalized to take account of within-subject differences (Loftus \& Masson, 1994). Strikingly, the relative beta values across the four metrical positions (Figure 3B) closely matched the pattern of RTs across the metrical positions (Figure 2), illustrating the correspondence between behavioral and neural effects. For both the auditory and visual modalities, con- ditions MP1 and MP8 had a greater effect on activity in the left IPC than conditions MP4 and MP5.

\section{Modality by Meter Interaction}

Despite the main effect of Target Modality in modalityspecific sensory cortices, the interaction between target modality and metrical position was not significant at the whole-brain level. Using the VOI approach showed a significant cluster of activation in the left IPC ([ $-32-47$ 56], $Z=3.99, p=.03 \mathrm{FWE}$ ) for the [(AuditoryStrong AuditoryWeak) - (VisualStrong - VisualWeak)] contrast, reflecting greater activation of this region for targets presented at strong metrical positions when targets were presented in the auditory as opposed to the visual modality (see Figure 3B).

\section{PPI Analysis}

In our PPI analysis, we analyzed changes in the regional connectivity between the left IPC and other brain regions (specified above) as a function of metrical position. Results revealed a broad network of functionally connected areas, with left and right Heschl's gyrus, left and right inferior occipital cortex, SMA, and cerebellum surviving the SVC for multiple comparisons (Table 2). BG, however, failed to survive statistical thresholding.

\section{DISCUSSION}

We examined brain activity associated with rhythmically induced cross-modal improvements in target detection.
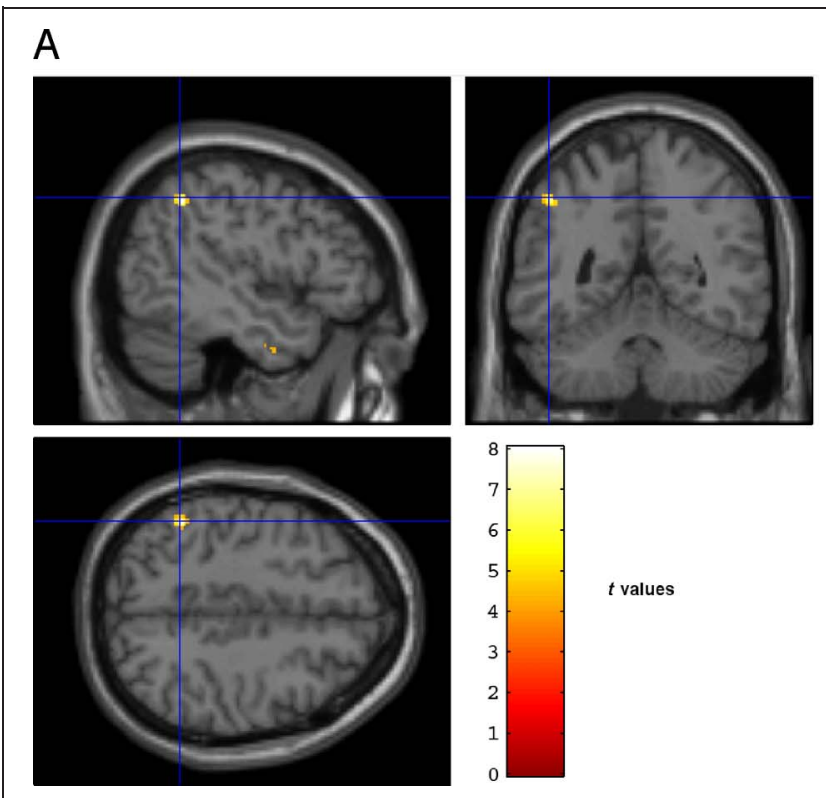

B

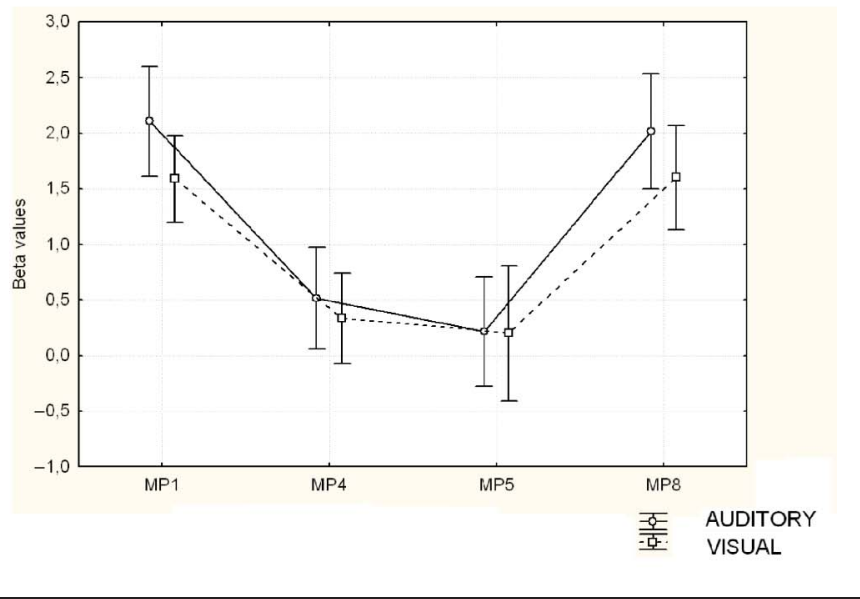

Figure 3. (A) Main effect of metrical position showing the left IPC $[-46,-50,44]$. (B) Beta values for the four different metrical positions averaged across participants for auditory and visual target detection tasks. 
Table 2. Areas of Activation as Showing Stronger Connectivity between Left IPC and VOIs at Strong Compared with Weak Metrical Positions (Both Auditory and Visual Modalities; * = Visual Modality Only; $\pm=$ Auditory Modality Only)

\begin{tabular}{|c|c|c|c|c|c|c|}
\hline Region & $x$ & $y$ & $z$ & $B A$ & $Z$ & $p_{\text {corrected }}$ \\
\hline SMA & -3 & 3 & 66 & BA 6 & 4.6 & .006 \\
\hline Right Heschl's gyrus and PT & 51 & -30 & 9 & BA 41 & 3.82 & .013 \\
\hline Left Heschl's gyrus and PT & -53 & -23 & 6 & BA 41 & 3.54 & .032 \\
\hline Right Heschl's gyrus and $\mathrm{PT}^{ \pm}$ & 62 & -36 & 18 & BA 42 & 3.78 & .014 \\
\hline Right inferior occipital cortex* & 33 & -84 & -2 & BA 19 & 3.36 & .07 \\
\hline Left inferior occipital cortex* & -54 & -72 & -6 & BA 19 & 3.88 & .017 \\
\hline Right cerebellum & 12 & -80 & -16 & - & 4.39 & .068 \\
\hline Left cerebellum & -27 & -60 & -27 & - & 3.62 & .001 uncorr \\
\hline Left putamen & -30 & -9 & 8 & & 2.92 & .002 uncorr \\
\hline
\end{tabular}

Participants were entrained to a simple auditory metrical sequence that lasted throughout the experiment and were asked to detect visual and auditory targets presented at various points within the metrical structure of the sequence. Behavioral responses were faster for targets presented on strong, as opposed to weak, beats, indicating increased allocation of attention to strong beats (see also Jones, 1985, 2010) and confirming previous behavioral results (Bolger et al., 2013; Escoffier et al., 2010). This behavioral benefit was accompanied by selective activation of left IPC. Moreover, the functional connectivity between left IPC and sensory cortices (visual and auditory), as well as SMA and cerebellum, was stronger when targets were presented on strong rather than weak beats.

One of the first points to be addressed is that when examining the effects of meter on brain activity we did not find structures typically associated with rhythm or beat processing, such as BG or premotor cortex (e.g., Grahn \& Brett, 2007; Chen et al., 2006). Our only observation of these structures was the subthreshold connectivity of the left putamen to the IPC in the PPI analyses. Although this might seem surprising, it must be remembered that the rhythmic stimulus used in our setup was highly regular and, most crucially, uninterrupted. Therefore, volunteers were most likely "feeling" the beat constantly throughout the entire experiment. Previous fMRI research in the field of beat and rhythm has compared rhythmically simple to complex or "nonrhythmic" stimuli (Geiser et al., 2012; Grahn \& Rowe, 2009; Grahn \& Brett, 2007) or varying levels of metrical saliency (Chen et al., 2006, 2008; Bengtsson et al., 2005). Here, by contrast, we did not compare sequences of varying rhythmicity but, instead, compared activity induced by strong versus weak beats within a continuously presented metrical rhythm. Critically, all experimental conditions were equally rhythmic to equate (or cancel out) brain activity related to rhythm and beat perception per se. When taking this into account, the lack of BG or premotor cortex activity becomes less surprising.
Indeed, our aim was to control for the fundamental process of perceiving rhythm and to focus, instead, on the effects of variations in beat saliency within a given rhythm on accompanying behavior. According to the DAT (Jones), stimuli presented at strong versus weak beats differ in terms of the amount of attentional energy allocated toward them. Strong beats capture attention, thus favoring the processing of any stimuli that happen to occur at those moments in time. Two recent fMRI studies have reported that the behavioral facilitation induced by rhythmic stimulus presentation was accompanied by increased activity in BG (Geiser et al., 2012) as well as bilateral pFC, insula, and left IPC (Marchant \& Driver, 2012). However, in both of these studies, rhythmic sequences were compared with nonrhythmic ones, thereby confounding basic perception of rhythm with the use of this rhythm to optimize behavior. In our study, we deliberately controlled for rhythm perception processes, thereby isolating activity related to beat-induced variations in the allocation of attention to precise moments in time more selectively. Our results indicate that, whereas BG and SMA may be activated by the perception of rhythm in the first place (e.g., Grahn \& Brett, 2007), the left IPC is activated whenever temporally salient elements of that rhythm capture attention, thereby optimizing the processing of stimuli occurring at that time. This neuroanatomical distinction is an example of the functional difference between timing to estimate duration (perceiving rhythmicity) and timing to optimize sensorimotor processing (using the temporal predictability of the rhythm to improve target detection; Coull \& Nobre, 2008).

\section{Implications for DAT}

Our main hypothesis was a greater involvement of cortical areas implicated in temporal orienting of attention for targets presented at strong compared with weak metrical positions. In particular, previous literature has shown a major role of the left IPC in temporal orienting 
(e.g., Coull \& Nobre, 1998, 2008). In previous temporal orienting studies using symbolic cues, timing was measured implicitly by the speed of motor responding or the accuracy of perceptual discrimination, although the way in which attention was oriented in time by the cues was explicit and voluntary. In the current study, by contrast, attention was oriented in an entirely implicit manner. Similarly, DAT proposes that metrically structured isochronous rhythms modulate attention in time in an automatic fashion. Our volunteers were given no instructions concerning the rhythm, and the target detection task did not require any reference to be made to rhythm or to time. Moreover, debriefing showed that none of the participants realized that targets appeared only around certain events in the trial. Consequently, participants were unaware of the independent variable being manipulated. Targets were presented at seemingly random intervals throughout a continuously present "background" auditory rhythm. However, despite the automatic nature of attentional allocation in this paradigm, strong metrical positions selectively activated the same area of the brain (i.e., left IPC) previously shown to be activated by the voluntary allocation of attention induced by symbolic temporal cues (Coull \& Nobre, 1998, 2008). This neuroanatomical overlap strongly suggests that the metrical structure of a rhythm automatically modulates orienting of attention in time, as predicted by the DAT. Importantly, although DAT was at first suggested as a theoretical framework (Jones, 1976), it has since received ample behavioral support. Previous studies have shown, for instance, smaller JND (just noticeable differences) or shorter RTs when judgments or responses were required at strong compared with weak positions. These studies have interpreted this speeding of RTs, or lowering of JND, in terms of facilitatory processing mediated by attention. Our results provide a neural complement to these behavioral findings by showing a direct involvement of the left IPC, the key structure in temporal orienting, in the performance benefits induced by a metrical auditory sequence. We feel our data lend substantial neurobiological support for the validity of DAT.

\section{Implications for Temporal Orienting of Attention}

In the spatial domain, a distinction has been drawn between exogenous and endogenous orienting of attention. Attention is said to be oriented exogenously when it is directed automatically to one particular side of space by the stimulus' physical salience (i.e., bottomup capture of attention). In contrast, attention is oriented endogenously when it is directed voluntarily by task instructions (i.e., top-down control of attention). A similar distinction can be drawn in the temporal domain (Coull \& Nobre, 2008): The inherent temporal structure of a rhythm is proposed to orient attention in a bottom-up, exogenous manner (e.g., Barnes \& Jones, 2000), whereas learned symbolic cues orient attention in a top-down voluntary manner (e.g., Coull \& Nobre, 1998). Although exogenous and endogenous mechanisms for orienting attention in space are functionally distinct they recruit overlapping neural networks (Kincade, Abrams, Astafiev, Shulman, \& Corbetta, 2005; Rosen et al., 1999; Nobre, Sebestyen, Gitelman, Mesulam, Frackowiak, \& Frith, 1997). Analogously, exogenous and endogenous mechanisms of orienting attention in time have been shown to be functionally distinct (Rohenkohl et al., 2011), with the current set of data further supporting the hypothesis (Coull \& Nobre, 2008) that their neural circuitry overlaps. Specifically, we have shown that rhythm-induced, exogenous temporal orienting selectively activates a region of left IPC that overlaps with the region activated by endogenous temporal orienting (Coull \& Nobre, 1998). The current data do not only confirm the context-independent role of the left IPC in temporal orienting but also extend its role to the auditory domain. Previous fMRI studies of temporal orienting have directed attention either endogenously with visual cues (Cotti et al., 2011; Davranche et al., 2011; Coull \& Nobre, 1998) or exogenously with visual trajectories (Coull et al., 2008; Assmus et al., 2003). Here, we show that the left IPC is also activated by temporal orienting when attention is directed by the metrical structure of an auditory rhythm.

Note that, although it is difficult to dissociate temporal orienting-related processes from target detection-related processes, particularly the difference in RTs between conditions, we believe that the left parietal activation is unlikely to simply reflect faster motor responses for two main reasons. First, it has previously been found that temporal orienting activates left parietal cortex not only independently of the type of motor response being executed (Cotti et al., 2011) but also independently of the need to make a speeded motor response at all: Specifically, temporal orienting activated left IPC even when the task for the participant was to make a nonspeeded perceptual discrimination rather than a speeded motor response (Davranche et al., 2011; Coull et al., 2008). Second, if left parietal activation simply reflected faster RTs, then one would expect to find this activation whenever there are differences in RTs, independent of whether one is interested in temporal orienting, memory, or perceptual processing, which is clearly not the case in the fMRI literature. Nevertheless, future studies and analyses should attempt to dissociate temporal orienting processes from target detection ones.

Although targets presented just before the strong beat might be thought to induce a weaker level of temporal expectancy (Figure 1) than those presented exactly on the beat, we found that targets presented at MP8 were detected just as well as those presented at MP1. This is an important finding insofar as it shows that participants were not simply reacting to targets presented at MP1 but were rather anticipating it. Similarly, targets presented just before the weak midbeat (MP4) were detected no differently to those presented on the midbeat (MP5). These data replicate the behavioral findings of Bolger et al. 
(2013), who used both the speeded detection paradigm used in the current study as well as a perceptual discrimination version of the same task. This is an important point because our previous results were obtained independently of the acoustic and musical structure of the entraining stimulus, that is, similar results were found for classical music recordings, in which tones on strong or weak beats were acoustically similar, as for a metronome. This refutes the possibility that the attentional entrainment observed in the current study is driven simply by the acoustic or physical saliency of the MP1 tones.

Coming back to the similarity of results for targets presented at MP1 and MP8 on one side and MP and MP4 on the other, one explanation is that there is a temporal "window" of expectancy, and events falling within this window are subjected to the same attentional benefits as events occurring precisely at the expected moment. Indeed, Jones and colleagues proposed a temporal gradient of attention, with benefits for temporal (Barnes \& Jones, 2000) or even nontemporal (Jones et al., 2002) discrimination performance decreasing the farther an event was presented from an entrained moment in time. Similarly, using a speeded detection task, Piras and Coull (2011) observed a U-shaped profile of attentional benefit centered around an expected moment in time: RTs were fastest for targets appearing at, or close to, the entrained moment in time but became progressively slower the earlier (or later) they appeared from the expected time. Future experiments could try to quantify the size of this window relative to the expected duration, although it is likely that its size would vary as a function of stimulus characteristics and task requirements. For example, in the auditory discrimination paradigm used by Jones et al. (2002), performance benefits were as strong for targets presented within $24 \mathrm{msec}$ of the entrained 600-msec interval as for those presented precisely at $600 \mathrm{msec}$, making the temporal window of expectancy approximately $4 \%$ of the entrained interval. On the other hand, in the visual speeded detection task of Piras and Coull (2011), benefits for probes presented within $100 \mathrm{msec}$ of the $600-\mathrm{msec}$ expected interval or within $300 \mathrm{msec}$ of the 1400-msec expected interval were no different to those presented exactly at the expected interval, making the temporal window of attention in this paradigm somewhat larger, $17-21 \%$ of the expected interval. Very similar effects were seen in another visual detection paradigm (Griffin, Miniussi, \& Nobre, 2001), in which benefits equivalent to those seen at the expected interval were observed for probes presented within $100 \mathrm{msec}$ of a 600-msec cued interval or within $50 \mathrm{msec}$ of a 300-msec cued interval, again making the temporal window of attention 17\% of the cued interval. In the current auditory detection paradigm, we found behavioral facilitation for targets presented $250 \mathrm{msec}$ before a 2000-msec entrained period, yielding a temporal attentional window that was slightly smaller, of around $12 \%$. However, until a systematic investigation of the putative window of attention has been undertaken (e.g., keeping the expected interval and probe ranges constant across paradigms), it is difficult to usefully compare across visual/auditory modalities or discrimination/detection paradigms.

\section{Intramodal and Cross-modal Effects}

By positing that metrical rhythmic structure induces attentional modulations in time, DAT makes the prediction that these attentional modulations should affect stimulus processing independently of modality. In this respect, there are at least two different ways of looking at the multimodal aspects of DAT. A first approach is to examine whether entrainment and changes in attentional energy can be transferred between different modalities, for instance auditory and visual. Recently, Grahn et al. (2011) showed that, although visual sequences are less effective than auditory sequences in inducing a feeling of beat, a stronger sense of beat could be generated by preceding visual sequences with auditory sequences of a similar temporal structure. This was accompanied by increased activity in BG (putamen) compared with visual sequences without prior auditory exposure. These results demonstrate the supremacy of the auditory modality in generating beat perception (see also Grondin \& McAuley, 2009; Repp \& Penel, 2002) but also, intriguingly, provide evidence of cross-modal influences in temporal attention (see also Lange \& Röder, 2006).

A second way of considering the multimodal properties of DAT is to examine whether entrainment in one modality (e.g., auditory) can affect processing in two, cooccurring modalities (e.g., auditory and visual). In other words, whether, once oscillations in attentional energy are entrained by a metrical structure, changes in the system are limited to the modality that has engendered entrainment-a sort of auditory dynamic attending - or whether they also extend to other modalities. Answering this question would address the generalization of DAT as well as the amodal nature of temporal attention. This is the approach we chose to implement here, based on previous behavioral studies showing that attentional entrainment by an auditory meter can benefit processing of visual stimuli when these were presented in, rather than out of, synchrony with the background auditory meter (Bolger et al., 2013; Brochard, Tassin, \& Zagar, 2013; Miller, Carlson, \& McAuley, 2013; Escoffier et al., 2010). Moreover, using both speeded detection and discrimination versions of the task, we further demonstrated that the effects of meter on performance were independent of the modality of the target and were induced as effectively by a simple metrical structure, like a metronome, as a more ecological stimulus, such as a recording of classical music (Bolger et al., 2013). This is important insofar as it shows that the cross-modal effect of meter is not restricted to isochronous stimuli but is a general property of structured rhythms that applies equally to highly variable and more musical rhythmic patterns. 
First, our fMRI data confirmed that volunteers were processing auditory and visual targets as expected: The main effect of Modality revealed significant activation of the auditory cortex during the auditory tasks and of visual cortex during the visual tasks. However, these areas were not differentially activated as a function of metrical position: The Modality $\times$ Metrical Position interaction showed only subthreshold activations in primary sensory cortices, suggesting they were activated similarly by strong and weak beats. The only region that did show significant differential activity for strong versus weak beats as a function of modality was left IPC: Specifically, the increased activity in left IPC because of strong beats was greater when targets were presented in the auditory modality than in the visual modality. This differential effect is likely because of the stronger influence of intramodal as compared with cross-modal effects: The auditory rhythm oriented attention more strongly for targets appearing in the same modality (i.e., auditory) than for targets appearing in a different modality (i.e., visual), resulting in attentional benefits that tended to be stronger for auditory, rather than visual, targets. Interestingly, despite the fact that primary sensory cortices were not differentially activated by metrical position, connectivity between these areas and left IPC did change significantly as a function of metrical position. Specifically, the PPI analysis revealed that increases in left IPC activity during strong beats covaried with increases in auditory and visual cortices, as well as in SMA and cerebellum. Therefore, although the entraining rhythm was auditory, we found evidence of increased connectivity between activity in left IPC and early visual cortex as a function of beat strength. This is strongly suggestive of a cross-modal mechanism, mediated by meter-induced activity in left IPC. Although PPI analysis does not permit inferences concerning the direction of connectivity between structures, it is possible that left IPC modulates sensory processing in an oscillatory manner, mimicking the metrical structure, rendering target processing more or less efficient.

Finally, increased connectivity between left IPC and SMA and cerebellum may reflect the role both of these areas play in timing or, more precisely, estimation of stimulus duration. SMA has consistently been implicated in duration estimation, whether for perceptual or motor timing or for timing of sub- or suprasecond durations (Schwartze, Rothermich, \& Kotz, 2012; Coull, Cheng, \& Meck, 2011; Wiener, Turkeltaub, \& Coslett, 2010). Cerebellum, on the other hand, is activated more specifically by sequential, subsecond or motor timing (Coull et al., 2011; Teki, Grube, Kumar, \& Griffiths, 2011; Lewis \& Miall, 2003; Penhune, Zattore, \& Evans, 1998). Given that our paradigm requires motor responding to targets presented against a sequence of tones separated by 250 msec, covariation with cerebellum, as well as SMA, is not surprising. We may speculate that SMA and cerebellum are recruited to time the intervals between tones, with temporal information then being passed to
IPC, which, in turn, modulates the degree of attentional resources allocated to the timed interval so as to enhance its processing (see also Teki, Grube, \& Griffiths, 2012; Coull, 2011).

To conclude, the fMRI study presented here highlights the role of auditory meter in modulating attentional resources in time and supports the idea, in line with DAT, that enhanced performance at metrically salient moments is because of attention being oriented in time. In addition, our results suggest that the facilitatory effects of orienting attention in time are, to a certain degree, independent of modality, and, in so doing, paves the way for further investigation into the cross-modal characteristics of temporal orienting of attention by auditory rhythms.

\section{Acknowledgments}

This experiment was conducted thanks to the support of the French National Agency (ANR-07-NEURO-033-01). We thank Bruno Nazarian, Muriel Roth, and Jean-Luc Anton of the fMRI Centre of Marseille for technical help and Kia Nobre and three anonymous reviewers for helpful comments on a previous version of this manuscript.

Reprint requests should be sent to Daniele Schön, UMR1106, INSERM, 27 Boulevard Jean Moulin, 13005 Marseille, France, or via e-mail: daniele.schon@univ-amu.fr.

\section{REFERENCES}

Andersson, J. L. R., Hutton, C., Ashburner, J., Turner, R., \& Friston, K. (2001). Modelling geometric deformations in EPI time series. Neuroimage, 13, 903-919.

Ashburner, J. (2007). A fast diffeomorphic image registration algorithm. Neuroimage, 38, 95-113.

Assmus, A., Marshall, J. C., Ritzl, A., Noth, J., Zilles, K., \& Fink, G. R. (2003). Left inferior parietal cortex integrates time and space during collision judgments. Neuroimage, 20, 82-88.

Barnes, R., \& Jones, M. R. (2000). Expectancy, attention and time. Cognitive Psychology, 41, 254-311.

Bengtsson, S. L., Ehrsson, H. H., Forssberg, H., \& Ullén, F. (2005). Effector-independent voluntary timing: Behavioural and neuroimaging evidence. The European Journal of Neuroscience, 22, 3255-3265.

Bengtsson, S. L., Ullén, F., Henrik Ehrsson, H., Hashimoto, T., Kito, T., Naito, E., et al. (2009). Listening to rhythms activates motor and premotor cortices. Cortex, 45, 62-71.

Besle, J., Hussain, Z., Giard, M. H., \& Bertrand, O. (2013). The representation of audiovisual regularities in the human brain. Journal of Cognitive Neuroscience, 25 365-373.

Bolger, D., Trost, W., \& Schön, D. (2013). Rhythm implicitly affects temporal orienting of attention across modalities. Acta Psychologica, 142, 238-244.

Brochard, R., Tassin, M., \& Zagar, D. (2013). Got rhythm... for better and for worse. Cross-modal effects of auditory rhythm on visual word recognition. Cognition, 127, 214-219.

Büchel, C., \& Friston, K. J. (2002). Assessing interactions among neuronal systems using functional neuroimaging. Neural Networks, 13, 871-882.

Bueti, D., Bahrami, B., Walsh, V., \& Rees, G. (2010). Encoding of temporal probabilities in the human brain. Journal of Neuroscience, 30, 4343-4352. 
Bueti, D., \& Macaluso, E. (2010). Auditory temporal expectations modulate activity in visual cortex. Neuroimage, 51, 1168-1183.

Chen, J. L., Penhune, V. B., \& Zatorre, R. (2008). Listening to musical rhythms recruits motor regions of the brain. Cerebral Cortex, 18, 2844-2854.

Chen, J. L., Zatorre, R. J., \& Penhune, V. B. (2006). Interactions between auditory and dorsal premotor cortex during synchronization to musical rhythms. Neuroimage, 32, 1771-1781.

Correa, A., Lupiáñez, J., Madrid, E., \& Tudela, P. (2006). Temporal attention enhances early visual processing: A review and new evidence from event-related potentials. Brain Research, 1076, 116-128.

Correa, A., Lupiáñez, J., \& Tudela, P. (2005). Attentional preparation based on temporal expectancy modulates processing at the perceptual level. Psychonomic Bulletin and Review, 12, 328-334.

Cotti, J., Rohenkohl, G., Stokes, M., Nobre, A. C., \& Coull, J. T. (2011). Functionally dissociating temporal and motor components of response preparation in left intraparietal sulcus. Neuroimage, 54, 1221-1230.

Coull, J. T. (2011). Discrete neuroanatomical substrates for generating and updating temporal expectations. In S. Dehaene \& E. Brannon (Eds.), Time and number in the brain: Searching for the foundations of mathematical thought (pp. 87-101). London: Elsevier.

Coull, J. T., Cheng, R.-K., \& Meck, W. H. (2011). Neuroanatomical and neurochemical substrates of timing Neuropsychopharmacology, 36, 3-25.

Coull, J. T., Davranche, K., Nazarian, B., \& Vidal, F. (2013). Functional anatomy of timing differs for production versus prediction of time intervals. Neuropsychologia, 51, 309-331.

Coull, J. T., Frith, C., Büchel, C., \& Nobre, A. (2000). Orienting attention in time: Behavioral and neuroanatomical distinction between exogenous and endogenous shifts. Neuropsychologia, 38, 808-819.

Coull, J. T., \& Nobre, A. C. (1998). Where and when to pay attention: The neural systems for directing attention to spatial locations and to time intervals as revealed by both PET and fMRI. Neuroscience, 18, 7426-7435.

Coull, J. T., \& Nobre, A. C. (2008). Dissociating explicit timing from temporal expectation with fMRI. Current Opinion in Neurobiology, 18, 137-144.

Coull, J. T., Vidal, F., Goulon, C., Nazarian, B., \& Craig, C. (2008). Using time-to-contact information to assess potential collision modulates both visual and temporal prediction networks. Frontiers in Human Neuroscience, 2, 1-12.

Davranche, K., Nazarian, B., Vidal, F., \& Coull, J. T. (2011). Orienting attention in time activates left intraparietal sulcus for both perceptual and motor task goals. Journal of Cognitive Neuroscience, 23, 3318-3330.

Drake, C., Penel, A., \& Bigand, E. (2000). Tapping in time with mechanically and expressively performed music. Music Perception, 18, 1-23.

Dreher, J. C., Koechlin, E., Ali, S. O., \& Grafman, J. (2002). The roles of timing and task order during task switching. Neuroimage, 17, 95-109.

Escoffier, N., Yeo, D., \& Schirmer, A. (2010). Unattended musical beats enhance visual processing. Acta Psychologica, 135, 12-16.

Essens, P. J., \& Povel, D.-J. (1985). Metrical and nonmetrical representations of temporal patterns. Perception $\mathcal{E}$ Psychophysics, 37, 1-7.

Geiser, E., Notter, M., \& Gabrieli, J. D. E. (2012). A corticostriatal neural system enhances auditory perception through temporal context processing. Journal of Neuroscience, 32, 6177-6182.
Geiser, E., Zaehle, T., Jancke, L., \& Meyer, M. (2008). The neural correlate of speech rhythm as evidenced by metrical speech processing. Journal of Cognitive Neuroscience, 20, 541-552.

Grahn, J. A., \& Brett, M. (2007). Rhythm and beat perception in motor areas of the brain. Journal of Cognitive Neuroscience, 19, 893-906.

Grahn, J. A., \& Brett, M. (2009). Impairment of beat-based rhythm discrimination in Parkinson's disease. Cortex, 45, 54-61.

Grahn, J. A., Henry, M. J., \& McAuley, J. D. (2011). fMRI investigation of cross-modal interactions in beat perception: Audition primes vision, but not vice versa. Neuroimage, 54, 1231-1243.

Grahn, J. A., \& McAuley, J. D. (2009). Neural bases of individual differences in beat perception. Neuroimage, 47, 1894-1903.

Grahn, J. A., \& Rowe, J. B. (2009). Feeling the beat: Premotor and striatal interactions in musicians and non-musicians during beat processing. Journal of Neuroscience, 29, $7540-7548$.

Griffin, I., Miniussi, C., \& Nobre, A. (2001). Orienting attention in time. Frontiers in Biosciences, 6, 660-671.

Grondin, S., \& McAuley, J. D. (2009). Duration discrimination in crossmodal sequences. Perception, 38, 1542-1559.

Hutton, C., Bork, A., Josephs, O., Deichmann, R., Ashburner, J., \& Turner, R. (2002). Image distortion correction in fMRI: A quantitative evaluation. Neuroimage, 16, 217-240.

Jäncke, L., Loose, R., Lutz, K., Specht, K., \& Shah, N. J. (2000). Cortical activations during paced finger-tapping applying visual and auditory pacing stimuli. Brain Research. Cognitive Brain Research, 10, 51-66.

Jones, M. R. (1976). Time, our lost dimension: Toward a new theory of perception. Attention and Memory, Psychological Review, 83, 323-356.

Jones, M. R. (1985). Structural organization of events in time: A review. In J. A. Michon \& J. Jackson-Roy (Eds.), Time, mind and behavior (pp. 192-214). Heidelberg, Germany: Springer-Verlag.

Jones, M. R., \& Boltz, M. (1989). Dynamic attending and responses to time. Psychological Review, 96, 459-491.

Jones, M. R., Boltz, M., \& Kidd, G. (1982). Controlled attending as a function of melodic and temporal context. Perception \& Psychophysics, 32, 211-218.

Jones, M. R., \& Ralston, J. (1991). Some influences of accent structure on melody recognition. Memory and Cognition, 19, 8-20.

Jones, M. R., \& Yee, W. (1997). Sensitivity to time change: The role of context and skill. Journal of Experimental Psychology: Human Perception and Performance, 23, 693-709

Jones, M. R., Moynihan, H., MacKenzie, N., \& Puente, J. (2002). Temporal aspects of stimulus-driven attending in dynamic arrays. Psychological Science, 13, 313-319.

Karabanov, A., Blom, Ö., Forsman, L., \& Ullén, F. (2009). The dorsal auditory pathway is involved in performance of both visual and auditory rhythms. Neuroimage, 44, 480-488.

Kincade, J. M., Abrams, R. A., Astafiev, S. V., Shulman, G. L., \& Corbetta, M. (2005). An event-related functional magnetic resonance imaging study of voluntary and stimulus-driven orienting of attention. Journal of Neuroscience, 25, 4593-4604.

Klein, J. M., \& Jones, M. R. (1996). Effects of attentional set and rhythmic complexity on attending. Perception $\mathcal{E}$ Psychophysics, 58, 34-46.

Kung, S.-J., Chen, J. L., Zatorre, R. J., \& Penhune, V. B. (2013). Interacting cortical and basal ganglia networks underlying find and tapping to the musical beat. Journal of Cognitive Neuroscience, 25, 401-420. 
Lange, K., \& Röder, B. (2006). Orienting attention to points in time improves processing both within and across modalities. Journal of Cognitive Neuroscience, 18, 715-729.

Large, E. W. (2000). On synchronizing movements to music. Human Movement Science, 19, 527-566.

Large, E. W., \& Jones, M. R. (1999). The dynamics of attending: How we track time-varying events. Psychological Review, 106, 119-159.

Large, E. W., \& Palmer, C. (2002). Perceiving temporal regularity in music. Cognitive Science, 26, 1-37.

Lewis, P. A., \& Miall, R. C. (2003). Distinct systems for automatic and cognitively controlled time measurement: Evidence from neuroimaging. Current Opinion in Neurobiology, 13, 250-255.

Lewis, P. A., Wing, A. M., Pope, P. A., Praamstra, P., \& Miall, R. C. (2004). Brain activity correlates differentially with increasing temporal complexity of rhythms during initialisation, synchronisation, and continuation phases of paced finger tapping. Neuropsychologia, 42, 1301-1312.

Loftus, G. R., \& Masson, M. E. J. (1994). Using confidence intervals in within-subject designs. Psychonomic Bulletin and Review, 1, 476-490.

Marchant, J. L., \& Driver, J. (2012). Visual and audiovisual effects of isochronous timing on visual perception and brain activity. Cerebral Cortex, 23, 1290-1298.

Martens, S., \& Johnson, A. (2005). Timing attention: Cuing target onset interval attenuates the attentional blink. Memory \& Cognition, 33, 234-240.

Miller, J. E., Carlson, L. A., \& McAuley, D. J. (2013). Temporal allocation of visual attention when what you hear influences when you see: Listening to an auditory rhythm influences the temporal allocation of visual attention. Psychological Science, 24, 11-18.

Niemi, P., \& Naataanen, R. (1981). Foreperiod and simple reaction time. Psychological Bulletin, 89, 133-162.

Nobre, A. C. (2001). Orienting attention to instants in time. Neuropsychologia, 39, 1317-1328.

Nobre, A. C., \& Coull, J. T. (2010). Attention and time. Oxford: Oxford University Press.

Nobre, A. C., Sebestyen, G. N., Gitelman, D. R., Mesulam, M. M., Frackowiak, R. S., \& Frith, C. D. (1997). Functional localization of the system for visuospatial attention using positron emission tomography. Brain, 120, 515-533.

Parncutt, R. (1994). A perceptual model of pulse salience and metrical accent in musical rhythms. Music Perception, 11, 409-464.

Penhune, V., Zattore, R., \& Evans, A. (1998). Cerebellar contributions to motor timing: A PET study of auditory and visual rhythm reproduction. Journal of Cognitive Neuroscience, 10, 752-765.

Piras, F., \& Coull, J. T. (2011). Implicit, predictive timing draws upon the same scalar representation of time as explicit timing. PLOS One, 6, e1820.

Praamstra, P., Kourtis, D., Kwok, H. F., \& Oostenveld, R. (2006). Neurophysiology of implicit timing in serial choice reaction-time performance. Journal of Neuroscience, 26, 5448-5455.

Rao, S. M., Harrington, D. L., Haaland, K. Y., Bobholz, J. A., Cox, R. W., \& Binder, J. R. (1997). Distributed neural systems underlying the timing of movements. Journal of Neuroscience, 17, 5528-5535.

Repp, B. H., \& Penel, A. (2002). Auditory dominance in temporal processing: New evidence from synchronization with simultaneous visual and auditory sequences. Journal of Experimental Psychology: Human Perception $\&$ Performance, 28, 1085-1099.

Rimmele, J., Jolsvai, H., \& Sussman, E. (2011). Auditory target detection is affected by implicit temporal and spatial expectations. Journal of Cognitive Neuroscience, 23, 1136-1147.

Rohenkohl, G., Coull, J. T., \& Nobre, A. C. (2011). Behavioural dissociation between exogenous and endogenous temporal orienting of attention. PLOS One, 6, e1462.

Rohenkohl, G., \& Nobre, A. C. (2011). $\alpha$ oscillations related to anticipatory attention follow temporal expectations. Journal of Neuroscience, 31, 14076-14084.

Rosen, A. C., Rao, S. M., Caffarra, P., Scaglioni, A., Bobholz, J. A., Woodley, S. J., et al. (1999). Neural basis of endogenous and exogenous spatial orienting: A functional MRI study. Journal of Cognitive Neuroscience, 11, 135-148.

Rushworth, M. F., Nixon, P. D., Renowden, S., Wade, D. T., \& Passingham, R. E. (1997). The left parietal cortex and motor attention. Neuropsychologia, 35, 1261-1273.

Sakai, K., Hikosaka, O., Takino, R., Miyauchi, S., Nielsen, M., \& Tamada, T. (2000). What and when: Parallel and convergent processing in motor control. Journal of Neuroscience, 20, 2691-2700.

Schubotz, R. I., Friederici, A. D., \& von Cramon, D. Y. (2000). Time perception and motor timing: A common cortical and subcortical basis revealed by fMRI. Neuroimage, 11, 1-12.

Schwartze, M., Rothermich, K., \& Kotz, S. A. (2012). Functional dissociation of pre-SMA and SMA-proper in temporal processing. Neuroimage, 60, 290-298.

Teki, S., Grube, M., \& Griffiths, T. D. (2012). A unified model of time perception accounts for duration-based and beat-based timing mechanisms. Frontiers in Integrative Neuroscience, 5, 90.

Teki, S., Grube, M., Kumar, S., \& Griffiths, T. D. (2011). Distinct neural substrates of duration-based and beat-based auditory timing. Journal of Neuroscience, 31, 3805-3812.

Tzourio-Mazoyer, N., Landeau, B., Papathanassiou, D., Crivello, F., Etard, O., Delcroix, N., et al. (2002). Automated anatomical labeling of activations in SPM using a macroscopic anatomical parcellation of the MNI MRI single-subject brain. Neuroimage, 15, 273-289.

Wiener, M., Turkeltaub, P., \& Coslett, H. B. (2010). The image of time: A voxel-wise meta-analysis. Neuroimage, 49, 1728-1740.

Wiener, M., Turkeltaub, P. E., \& Coslett, H. B. (2011). Implicit timing activates the left inferior parietal cortex. Neuropsychologia, 48, 3967-3971.

Woodrow, H. (1914). The measurement of attention. Psychological Monographs, 17. 